lische Umwandlungsformen (Harries), sondern z. T. wenigstens als typische Oxydationsprodukte dieser Kohlenwasserstoffe anzusehen.

$$
\text { Nachtrag. }
$$

Die mittlerweile fortgeführten Untersuchungen haben ergeben, dab die Beschaffenheit der Reaktionsprodukte auferordentlich von folgenden Faktoren abhängig erscheint: 1 . Art des $Q u e l-$ lungsmittels, 2. Menge und vor allem $\mathrm{D}$ auer der Einwirkung der Konz. $\mathrm{H}_{2} \mathrm{SO}_{4}, 3$. Art des Kautschuks und Konzentration desselben in der Lösung.

So ergab, um nur ein Beispiel anzuführen, eine fünfprozentige Quellung eines afrikanischen Kautschuks (Ubanghi), in Schwefelkohlenstoff bei der Einwirkung von $5 \mathrm{ccm}$ konz. $\mathrm{H}_{2} \mathrm{SO}_{4}$ durch ca. 15 Minuten ein vollkommen rohgum miartiges Produkt, welches nur schwieriger und unvollständiger in $\mathrm{C}_{6} \mathrm{H}_{6}$ bzw. $\mathrm{CS}_{2}$ löslich war als das Ausgangsmaterial, während eine gleich- artige benzolische Lösung bei sonst gleicher Behandlungsweise und Dauer der Einwirkung ein amorphes, grünlich-weifes Produkt lieferte.

Der chemischen Umwandlung scheint demnach eine physikalische Veränderung des Kautschukkolloids voranzugehen, was auch durch die Aenderung der Viskosität der Lösungen nach Zugabe der $\mathrm{H}_{2} \mathrm{SO}_{4}$ angezeigt wird. Aulerdem scheint für die Geschwindigkeit der Umwandlung die Quellungsfähigkeit des, Lösungsmittels maßgeblich zu sein.

Während die Viskosität der $\mathrm{C}_{6} \mathrm{H}_{6}$ - Lösungen von der eintretenden Koagulation sehr rasch zurückgeht, ist bei der $\mathrm{CS}_{2}$-Lösung im Gegenteil sofort eine auffallende Erhơhung der ersteren $z u$ beobachten und erst nach längerer Dauer der Einwirkung $(1-2 \mathrm{St}$.) tritt allmählich eine Abnahme derselben ein. Die bei dieser längeren Einwirksdauer erhaltenen Produkte (aus der $\mathrm{CS}_{2}$-Lösung) sind ebenfalls bröcklig amorph und gleichen den oben beschriebenen.

Die Untersuchungen sollen auch in dieser Richtung fortgesetzt werden.

\title{
Ueber eine einfache Methode zur Herstellung von Ultrafiltern.
}

Von Erich Knaffl-Lenz.

(Eingregangen an 13 . Sejt, 1920.1

(Aus dem pharmakologischen Institut der Universitat Wien.)

Im folgenden sei eine Methode angegeben, die es ermöglicht, ohne besondere Apparatur rasch Kollodiumfilter für Ultrafiltrationen herzustellen. Da sich die Durchlässigkeit der Filter leicht variieren läbt, dürften sie für die meisten Fälle gute Dienste leisten ${ }^{1}$ ).

Erforderlich ist hierzu eine gewöhnliche geradwandige Porzellannulsche und eine ebensolche Kristallisierschale von genau dem gleichen Durchmesser. Durch Ausgießen der Kristallisierschale mit. Kollodium und vorsichtigem Drehen der Schale während des Ausgießens sorgt man, daß das Kollodium die Wände gleichmäBig benetzt und füllt nach Verdampfen des Aethers die Schale mit Wasser, welches im Verlaufe von zehn Minuten $2-3$ mal gewechselt wird. Das Kollodiumhäutchen; welches einen genauen AbguB der Schale darstellt, läBt sich nach Ablösen der Ränder leicht vom Glase entfernen, besonders dann, wenn man zwischen Membran und Glaswand langsam Wasser einflieBen läBt. Nun wird der Kollodiumsack in die feuchte, mit einem

1) Die von Wa. Ostwa ld (Koll,- Zeitschr. 22, 143, 1918 ) angegebene Methode wurde mir erst bei der Korrektur bekannt. gut passenden feuchten, nicht zu dünnen Filtrierpapier armierte Natsche so eingesetzt, dat er zentrisch sitzt and möglichst faltenfrei anliegt. Dies gelingt leicht, wenn der Kolloditumsack wahrend des Einsetzens teilweise mit Wasser gefüllt ist. Unter leichtem Ansaugen wird die Membran mit den Fingern an die Wände gepreßt. Falls die Kollodiumhaut nicht zu dick war und gut eingesetzt wurde, erhält man eine vollständige Abdichtung, so daß das durch die Pumpe erzielbare Vakuum voll zur Geltung kommen kann.

Die Durchlässigkeit der Filter für Kolloide läbt sich sowohl durch die Dicke der Filter als auch durch den Quellungsgrad des Kollodiums variieren. Die Kollodiumlösung soll hö̀chstens dreiprozentig sein, da konzentriertere Lösungen zu langsam fließen und ungleiche Dicke der Membran verursachen. Dickere Membranen stellt man zweckmäßig her durch zweimaliges Ausgieben der Schale mit 2-3 Proz. Kollodiumlösungen. Für die Permeabilität ist der Wassergehalt des Kollodiums von wesentlicher Bedeutung. LäBt man das Wasser auf däs Kollodium einwirken, nachdem der größre Teil des Aethers verdampft 
ist, so nimmt dasselbe viel mehr "Vasser auf, als wenn man den. Aether vollständig und den Alkohol teilweise verdampfen häbt. Die wasserreichen Membranen erscheinen opaleszenter und sind wesentlich durchlässiger. Die Filter müssen unter Wasser aufbewahrt werden, da sie sonst durch Schrumplung rasch unbrauchbar werden.

Mit derart hergestellten Ultrafiltern von $7 \mathrm{~cm}$ Durchmesser konnten im Laufe einer Stunde $600-700 \mathrm{ccm}$ eines Goldsols abgenutscht werden. Die dünnen und wasserreichen Filter ließen einen Teil des Goldsols durch, wahrend die dickeren und wasserärmeren das Gold vollständig zurückhielten. Ein geringer Zusatz von Serumeiweib, welches zur Verhinderung der Ooldfallung mit wenig Salz oder Salzsăure versetzt war'in, bewirkte auch bei dünnen wasserreichen Filtert ein vollstăndiges Zurückhalten des Goldsols auf dem Filter.

Handelt es sich um kleine zu filtrierende Mengen, so läht sich zweckmähig ein Goochtiegel in analoger Weise adaptieren. Die Herstellung des Kollodiumsäckchens erfolgt in einem in Größe und Form identischen Porzellanoder Metalltiegel.

3) Fo Kalfl-Lenz, Beitrag zur Kenntnis der Goldsole, erscheint spăter in dieser Zeitschrift,

\title{
Der Kautschuk im Jahre 1919. Jahresbericht über die Fortschritte der Kautschukchemie im Jahre 1919.
}

\author{
Von E. Fontobert (Wiesbaden).
}

Bei der Durchsicht der seit Kriegsende bis zum Ende des Jahres 1919 erschicnenen Arbeiten auf dem Gebiete des Kautschuks macht sicl noch wie in den vorhergehenden Jahren der Einfluf des Krieges sehr deutlich geitend. Neben einer'schr geringen Anzah! rein chemischer und kenstitutionsaufklärender Arbeiten findet sich eine große Zahl von Veröffentlichungen, die die praktische Verwertbarkeit und die Untersuchung der mehr technischen Verfahren und Produkte zum Ziel hat, daneben atch eine beträcittliche Anzahl von Arbeiten, die sich mit der Analyst des Kautschuks befassen, und dic weitgehend auf die Kriegsverhältnisse und ihre Ersatzstoffe Riicksicht nehmen. Aus den einzelnen $Z$ weigen des umfangreichen Gebietes moggen folgende Arbeiten Erwähnung finden.

\section{A, Kautsehukpflanzen und ilire Kultur.}

St af ford Whitby') unterzog such der Mühe, eine gewaltige Anzahl Băune von Hevea brasiliensis auf ihren Kătschukgehalt zu untersuchen. Neben anderem fand er, daß die Individualität des Baumes die gröbte Rolle spięt, und daB ein guter Baum mit einer hohen Ausbeute auch lange ein guter Baum bleibt. Dasselhe gilt auch tmgekehrt. Die Ausbeute selbst bestimmte er zu 0-27 g Kautschuk am Tag. $4 \mathrm{~g}$ kommen am meisten vor; der Durchschnitt liegt bei $7,12 \mathrm{~g}$. Als höchste Ausbeuten am Tage wurden einmal 41,45 und einmal $42,77 \mathrm{~g}$ gefunden. -Alle Untersuchungen deutèn daranf hin, dab eine Verbesserung der Kautschukausbeuten am meisten Erfolg durch eine zweckmainige Auswahl der Samen hat. Die Samen eines bestimmten Baumes sind gar nicht so schwer zu sammeln, da- sie sich meistens durch eine ganz individuelle Zeichnung von allen Samen der anderen Bäume unterscheiden.

Der Lmfang der Bäume steht in einem gewissen Verhältnis zur Kautschukausbeute. Außer der Ausbeute bleiben auch andere Eigenscliaften eines Baumes lange Zeit oder dauernd erhalten. Durch die auftretenden

s) Stafford Whitby, India Rubber Ioum 58, 895 (1919); C. 1920. II, 242 . starken Verschiedenheiten sind alle Versuche als $z$ weifelliaft $z u$ bętracthten, dic sich nur auf eille kleinere Anzahl von Baumen stitzen.

Die Notwendigkeit dor 'steten Eneuerung der Katitsclukpiantagen betrachtet Gordon Stanley Reis"), Ei kommt bei der Bestimmung der "Rotation", d. i. der Zeit, die zwischen dem Pfianzen eines Feldes mit Kautschukbãumen und dem Zeitpunkt liegt, zu dem ein Fallen und Neupflanzen der Bäume notwendig wird, da die alten Bäume keinen Ertrag mehr liefern, $z u$ dern Resultat, daß dieser Zeitraum aul 30 Jante festzusetzen ist.

Bubill of f hatte die Ansicht vertreten, daB die Blätter der Kautschukbäume keine-Beziehungen zum Milchsaft und dessen Menge hätten. Dieser Anschaung wird widersprochen ${ }^{3}$, unter anderem mit dem Argument, daB die Blätter so wichtige Faktoren im Leben eines Baumes sind, daß letzten Endes sie nicht ohne jede Beziehuitg zum Milchsaft sein kömnen.

Von den verschiedenen Kautschuksorten steht in der Kultur der Parakautschuk weiterhin an erster Stelle. Lediglich die Not, wie $z$. B. der Schiffsraummangel, haben stellenweise die Kultur anderer Pflanzen begünstigt. So gibt A n d rew $\mathrm{H}$. K in $\mathrm{g}^{4}$ ) Anleitung zur rationellen Kultur von Guayulekautschuk.

Wesentliche Anstrengutigen sind in der Erforschung und-Bekämpfung von Krankheiten und Schädingen der Kautschukbäumc gemacht worden. Neben Ameisen, die man an besten durch Ausräuchern vernichtet ${ }^{5}$ ), sind es besonders die Black-stripe und Brownbast genannten, durch Pilze hervorgenfenen Krankheiten, die den Farmern viele Sorge bereiten. W. N. C. Bel-

") Gordon Stan ley Reis, India Rubber Journ. 57, $14,19(1919) ;$ C. 1919, IV, 669.

) India Rubter Journ. 57, 120-(1919); C. 1920 , II, 134 .

4). Andrew H. King, Chen. Metall. Eng. 19, 23 $(1918) ;$ C. $1920, \mathrm{Il}, 132$ 1. $18 \%$ 